\title{
Digital Image Correlation Data Processing and Analysis Techniques to Enhance Test Data Assessment and Improve Structural Simulations
}

\author{
Nathaniel W. Gardner ${ }^{1}$, \\ Analytical Services \& Materials, Inc., Hampton, VA, 23666 \\ Mark W. Hilburger ${ }^{2}$, Waddy T. Haynie ${ }^{3}$, Michael C. Lindell ${ }^{4}$, \\ NASA Langley Research Center, Hampton, VA, 23681 \\ W. Allen Waters ${ }^{5}$ \\ Analytical Mechanics Associates, Hampton, VA, 23666
}

The NASA Shell Buckling Knockdown Factor Project (SBKF) was established in 2007 by the NASA Engineering and Safety Center (NESC) with the primary goal to develop new analysis-based buckling design factors (a.k.a. knockdown factors) and high-fidelity buckling simulations for selected launch-vehicle-like cylindrical shell structures. A series of tests are being conducted on large-scale metallic and composite cylindrical shells in order to provide validation data for these new factors and simulations. However, the validation of these new factors and simulations is quite demanding and requires test data that is commensurate with their fidelity. Traditional instrumentation, such as linear variable displacement transducers (LVDTs) and electrical-resistance strain gages serve a critical role in providing accurate displacement and strain measurements in these tests, but only allow for data to be recorded at a select number of point locations and are not sufficient to provide all the necessary validation data. Advanced measurement technologies can be used effectively to complement traditional instrumentation and gather additional data required to validate these structural simulations. In particular, three-dimensional digital image correlation (DIC) was implemented during SBKF cylinder testing to characterize the full-field displacement and strain behavior. Commercially available VIC-3D ${ }^{\mathrm{TM}}$ software and user-written data processing scripts were used to generate valuable data and insight into the complex buckling response of the cylinders that otherwise would be impossible to gather using traditional instrumentation. In addition, the measured data from DIC was used to verify measured test data obtained from other instrumentation, enhance test and analysis correlation, and help identify the root cause of anomalous test results that may have gone unexplained if only traditional instrumentation was used. Selected test results that demonstrate the use of DIC on the SBKF cylinders are presented and a portion of the data processing methods are described.

\section{Nomenclature}

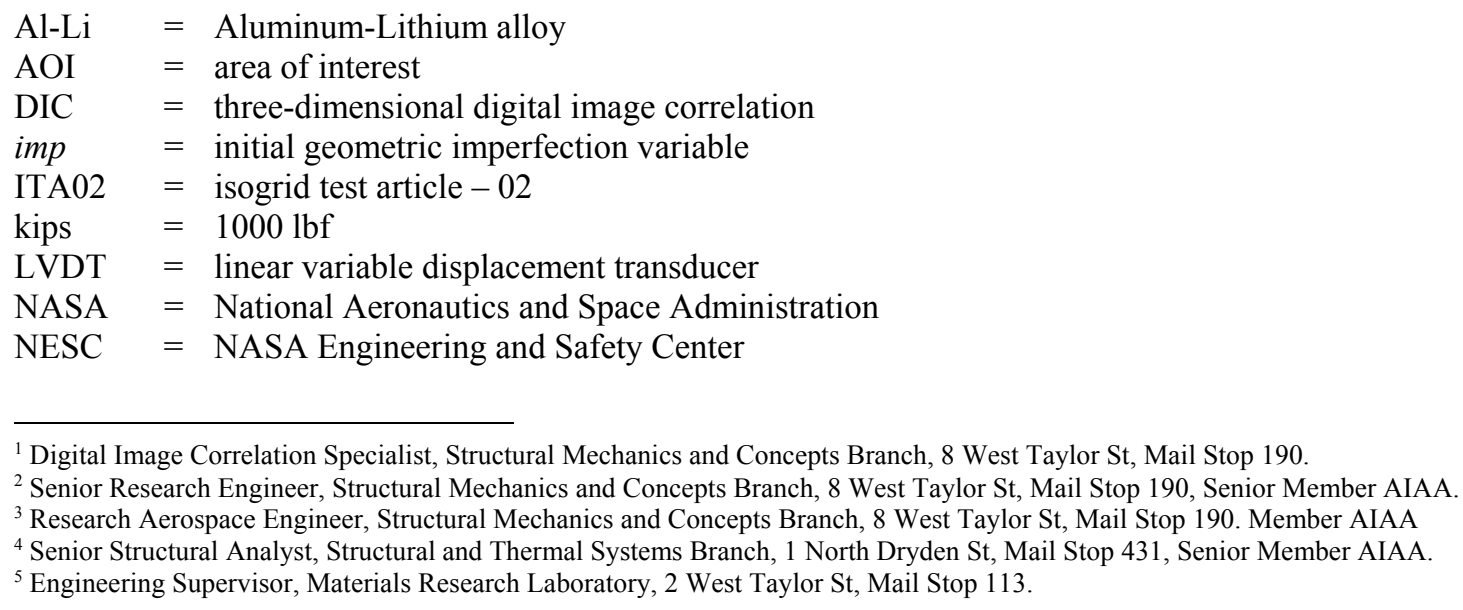




$\begin{array}{ll}\text { OML } & =\text { outer mold line } \\ \text { SBKF } & =\text { Shell Buckling Knockdown Factor Project } \\ R_{D I C} & =\text { DIC-based best-fit cylinder radius for a fully assemble cylinder data set } \\ R_{\text {measured }} & =\text { DIC-based radius measurement } \\ R_{V I C} & =\text { DIC-based best-fit arc radius for a single DIC system } \\ \mathrm{TA} 07 & =\text { orthogrid test article }-07 \\ u, v, w & =\text { DIC measured displacements in the } x, \theta, \text { and } z \text { directions, respectively } \\ x, \theta, z & =\text { global cylinder surface coordinates } \\ \Delta i m p & =\text { difference plot variable for initial imperfection, } i m p \\ \Delta u & =\text { difference plot variable for axial displacement, } u \\ \Delta w & =\text { difference plot variable for radial displacement, } w \\ \sigma_{\text {mag }} & =\text { DIC measurement uncertainty magnitude }\end{array}$

\section{Introduction}

The NASA Shell Buckling Knockdown Factor Project (SBKF) was established in 2007 by the NASA Engineering and Safety Center (NESC) with the goal to develop new analysis-based buckling design factors (a.k.a. knockdown factors) and high-fidelity buckling simulations for selected launch-vehicle-like cylindrical shell structures ${ }^{1}$. As part of this effort, a series of large-scale buckling tests have been conducted on metallic and composite cylindrical shells in order to provide data necessary to validate these design factors and buckling simulations. The validation of these new factors and simulations requires test data that accurately quantifies global and local behavioral characteristics of the cylinders, including displacements and strains during the prebuckling, buckling, and postbuckling ranges of loading. Traditional instrumentation, such as linear variable displacement transducers (LVDTs) and electrical-resistance strain gages, serve critical roles in providing accurate displacement and strain measurements in these tests. For example, displacement and strain measurements were used to monitor load introduction into the test article and strain measurements were used to characterize load distribution in complex built-up stiffened structures. However, this traditional instrumentation can only be used to collect data at discrete locations and results in very sparse coverage when applied to large-scale test articles. In addition, buckling of thin-walled cylinders typically exhibit complex and non-uniform behavior and can be very sensitive to variations in the as-tested cylinder geometry and loading conditions, which can make choosing measurement locations problematic. In particular, finite-element-based pretest predictions are often used to help define instrumentation needs and locations and typically assume idealized test conditions (e.g., loads and boundary conditions). However, differences between the actual test conditions and the corresponding pretest analysis assumptions are inevitable and can have a significant influence on the structural response and the usefulness of some of the instrumentation when capturing specific local response characteristics. These instrumentation challenges, along with practical considerations associated with the instrumentation of large-scale structural test articles, limit the ability of traditional measurement techniques in providing all the necessary data for the SBKF validation activities. However, advanced full-field measurement technologies such as three-dimensional digital image correlation, referred to hereafter as DIC, can be used to complement traditional instrumentation and gather the additional data required to meet the SBKF validation data requirements.

To date, ten subscale 8-ft-diameter cylinder buckling tests (nine metallic cylinders and one sandwich composite cylinder) and two full-scale 27.5-ft-diameter metallic cylinder buckling tests have been conducted. DIC has been used, along with traditional instrumentation, on all of the cylinder tests to gather full-field displacement and strain data, and has helped alleviate the aforementioned instrumentation challenges. The DIC data obtained from these tests has been used to refine and validate buckling simulations (see Ref. 2) and has helped identify and characterize anomalous behavior observed during one of the tests.

Selected DIC results from two different subscale metallic cylindrical test articles are presented herein and used to illustrate the application of DIC in large-scale structural testing and finite-element-model development. In particular, DIC data is presented and used to identify and characterize changes in the geometry of one cylinder test article that resulted from the installation of the test article into the test facility. DIC data from a second cylinder test is presented and used to identify the root cause of some anomalous test results. In both cases, the DIC results are then used to guide the development and refinement of finite-element-models to more accurately simulate the test behavior. The results from these refined models are also presented and compared to the corresponding test results.

A description of the cylindrical test articles and the subscale test facility are given in Section II. A detailed description of the DIC data processing is presented in Section III and includes data processing methods and userwritten data processing scripts used. Selected test results, test-analysis correlation, and finite-element-model refinements using the measured DIC data are presented in Section IV. 


\section{Test Overview}

Nine different subscale, 8-ft-diameter, 6-ft-tall, integrally-stiffened metallic cylinder test articles were designed, fabricated, and tested by SBKF. Each test article was constructed from three integrally-stiffened, 2195 AluminumLithium (Al-Li) curved-panel segments $\left(120^{\circ}\right.$ arc segments) that were friction-stir welded together along axial weld lands to form a complete cylinder. The stiffener configurations were representative of modern launch vehicle cylinder designs and included internal orthogrid and isogrid stiffener patterns. The orthogrid stiffener pattern and isogrid stiffener pattern are shown in Fig. 1. Once fabrication was complete, each end of the test article was potted in a singlepiece steel attachment ring using the low-melting-point alloy, Cerrobend. An internal view of a typical orthogridstiffened test article with attachment rings installed is shown in Fig. 2 and the cross-section of the test article and attachment ring interface is shown in Fig. 3. Additional details on these 8-ft-diameter metallic cylinders are presented in Ref. 2.

A special-purpose self-reacting test apparatus was designed and fabricated for the SBKF test effort, and is shown in Fig. 4. The test apparatus was comprised of two loading spiders, load struts, two load introduction cylinders, and eight load lines. Each of the load lines connect the upper and lower loading spiders and consisted of a hydraulic actuator, a 4-in-diameter loading rod, and a load cell. The 8-ft-diameter test article was attached between the upper and lower load introduction cylinders via single-piece attachment rings, and the load was applied to the cylinder by extending and contracting the hydraulic actuators in the load lines. A multi-channel load control system was used to apply the test loads. Strain and displacement data was obtained from over 300 locations on each test article using traditional electrical-resistance strain gages and LVDTs. In addition, full-field displacement and strain data was obtained on the outer surface (a.k.a. outer mold line or OML) of the test articles by using low-speed and high-speed DIC systems.

DIC is a non-contact optical technique used to measure three-dimensional shapes and deformations of structures. A single DIC system utilizes a pair of synchronized digital cameras to record minute movements or changes of a highcontrast speckle pattern applied to the surface of the test article. Prior to testing, the camera pair was calibrated using a known calibration target to determine the relative location of the two cameras in space, and other camera properties necessary for accurate image correlation. During testing, synchronized image pairs were recorded by the DIC cameras at a specified rate. The resulting images were analyzed using VIC-3D ${ }^{\mathrm{TM}}$ software, developed by Correlated Solutions, Inc. to evaluate the changes in the speckle pattern images, determining the full-field displacements and strains of the structure.

During a typical subscale cylinder buckling test, SBKF utilizes multiple low-speed and high-speed DIC systems positioned around the circumference of the test article to record images of the quasi-static and dynamic displacement and strain response of the visible outer surface of the cylinder. However, for the purpose of this paper, only the lowspeed DIC system and results will be discussed in detail. Prior to installing the cylinder into the test fixture, the outer surface of the cylinder was prepared with a high-contrast speckle pattern using a four-step process. First, the cylinder OML was painted with a flat white base coat. After the base coat was applied, the cylinder was wrapped with an adhesive-backed vinyl stencil consisting of randomly distributed oval-shaped cutouts used to generate the speckle pattern. The diameter of the speckles ranged from $0.45 \mathrm{in}$. to $0.70 \mathrm{in}$. and was calculated based on the spatial resolution of the DIC systems to ensure the highest resolution and accuracy was achieved during a given test. Once the stencil was applied to the outer surface of the cylinder, the cylinder OML and stencil was painted black. Finally, the stencil was removed to reveal a randomly distributed array of black speckles. Selected strain gage locations and coordinate reference locators were marked with a ' + ' symbol. The coordinate reference locators were positioned at the center of each panel and at the mid-length of each weld land. These markings were used in several ways during data processing and will be discussed in the upcoming sections. 


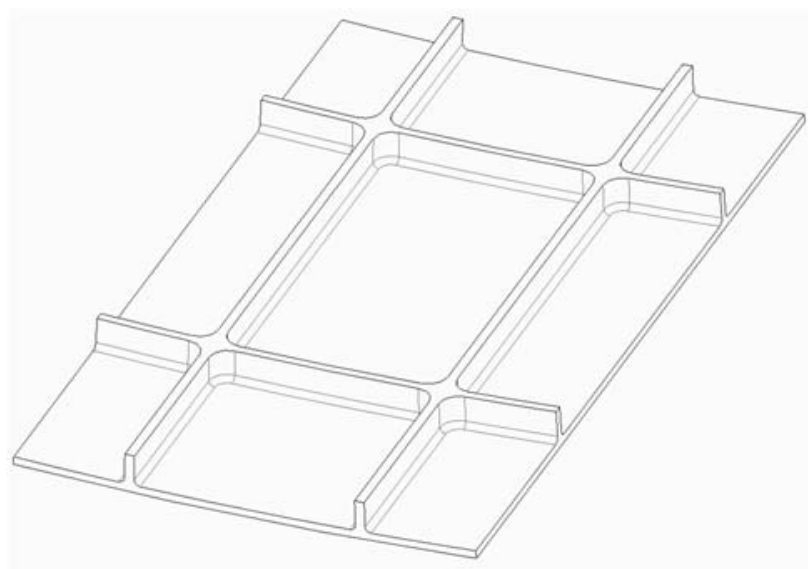

a) Orthogrid stiffener pattern

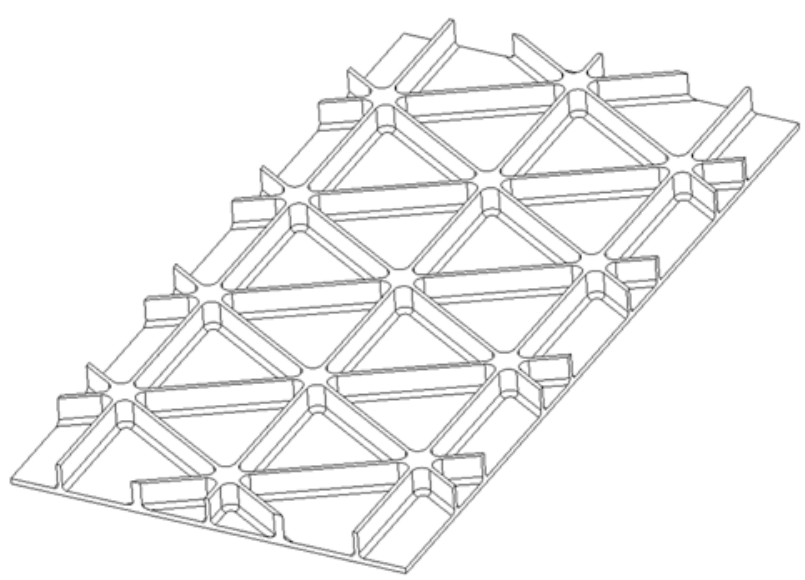

b) Isogrid stiffener pattern

Figure 1. Orthogrid and isogrid stiffener patterns.

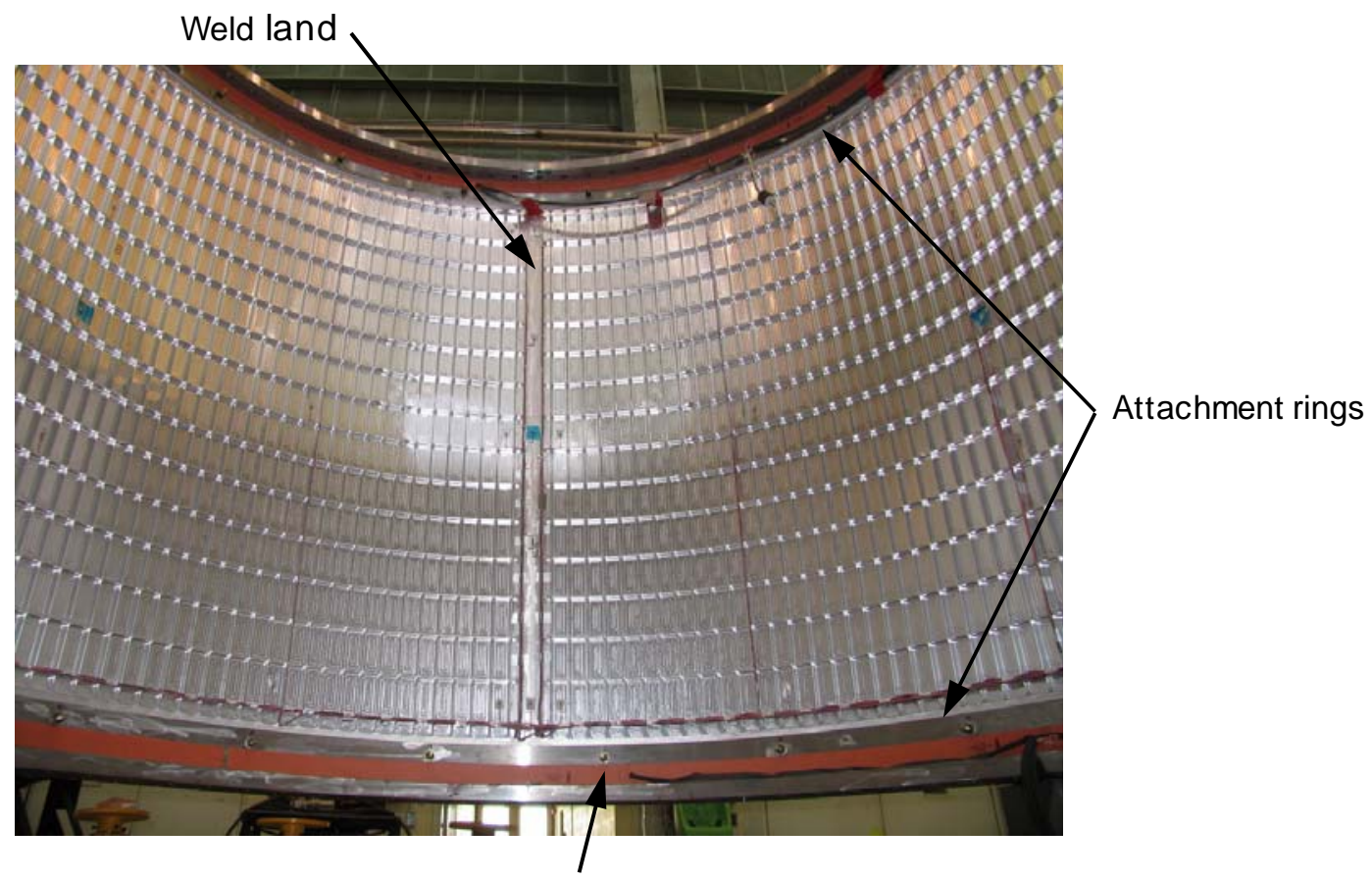

Fail-safe bolt (typ.)

Figure 2. Internal view of a typical orthogrid-stiffened cylinder test article, including a longitudinal weld land and attachment rings. 


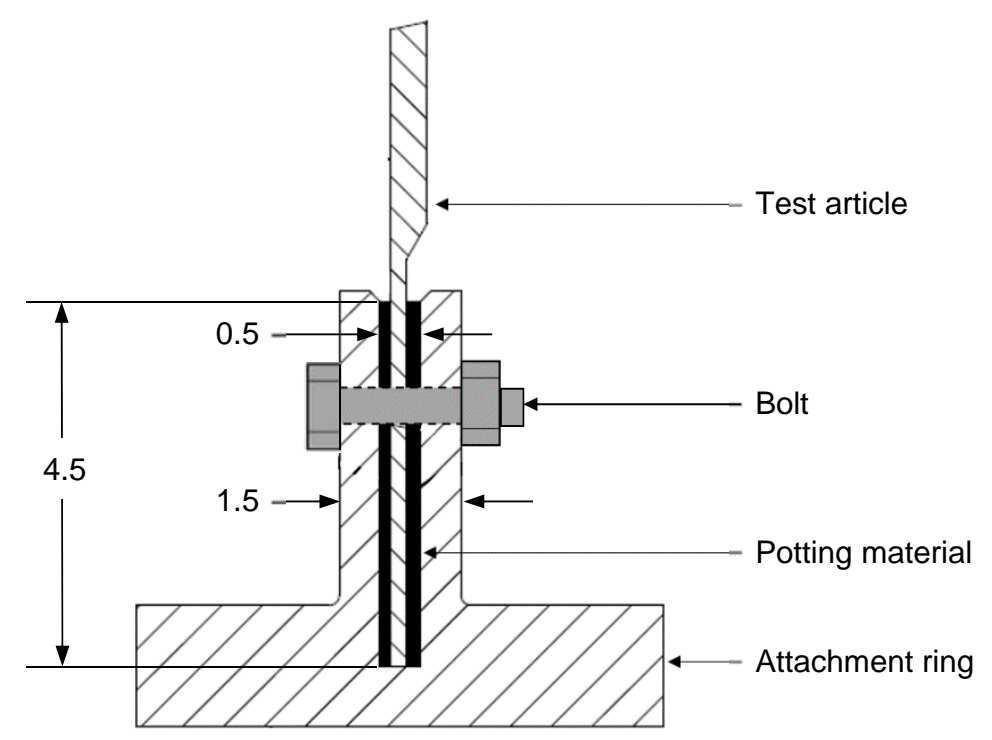

Figure 3. Attachement ring cross-section (dimension are in inches).

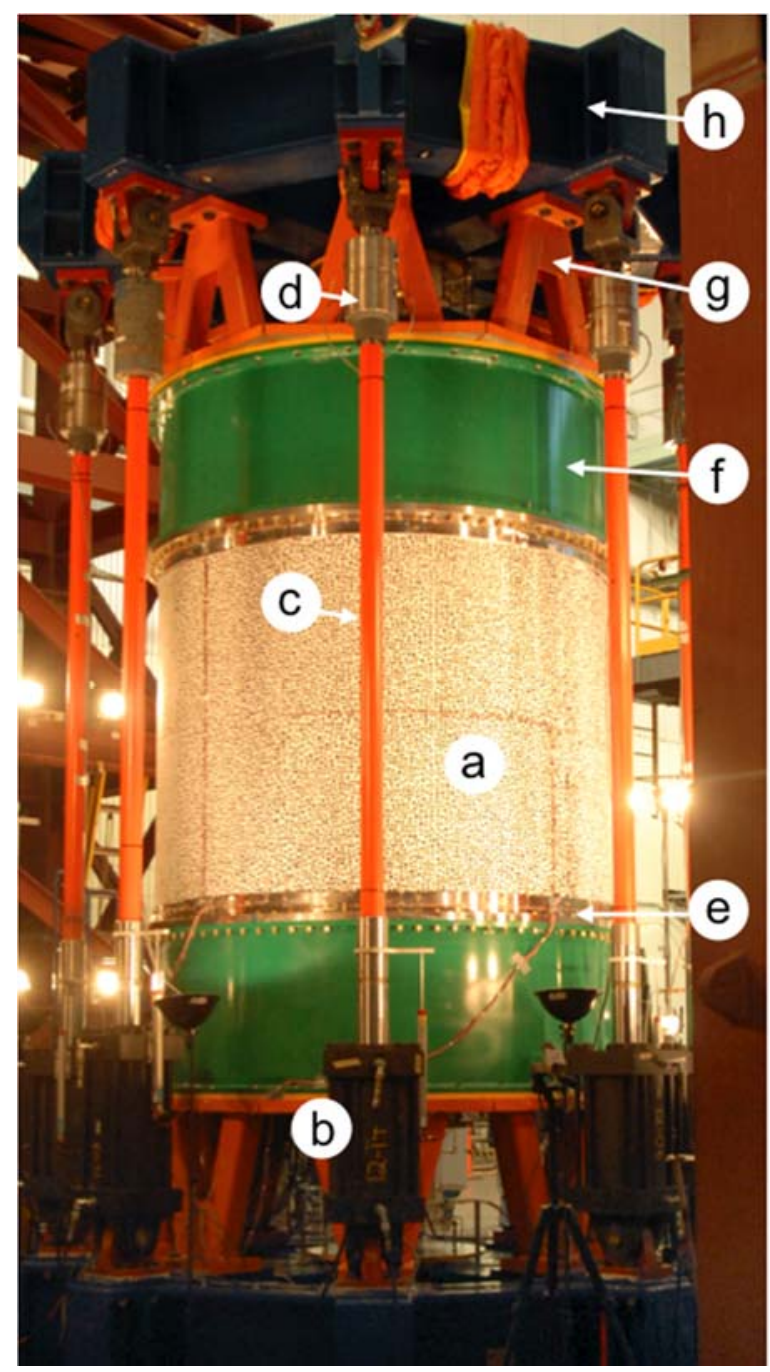

Figure 4. 8-ft-diameter shell buckling test facility: (a) test article, (b) hydraulic actuator, (c) loading rod, (d) load cell, (e) attachment ring, (f) load introduction cylinder, g) load strut and h) loading spider. 
A notional DIC system layout from a cylinder buckling test is shown in Fig. 5. In the figure, the cylinder test article cross-section is represented by a thick black circle, and the centers of each panel and weld land are denoted with hash marks. The load lines are denoted by the orange filled circles. The DIC systems are indicated by the gray and black camera icons, and the approximate fields of view for each system are represented by the lines emanating from the camera icons. Eight low-speed DIC systems were positioned approximately every $45^{\circ}$ around the circumference of the test article. Each system can correlate $60^{\circ}$ to $80^{\circ}$ arc-sections around the circumference of the cylinder and results in sufficient system-to-system image overlap to ensure there were no circumferential gaps when assembling the data from all systems (data assembly to be discussed in Section III).

Each DIC system used two 5-megapixel (2448 by 2048 pixels) cameras. The cameras in a system were mounted on a vertical bar (i.e., a bar aligned with the cylinder axis), so both cameras viewed the same circumferential span. The cameras were also rotated so the direction with the maximum pixel resolution was aligned with the cylinder axis. Cameras from all systems were synchronized using an external signal, and images were acquired at a rate of $0.2 \mathrm{~Hz}$. Images collected using the DIC systems were processed using the VIC-3D ${ }^{\mathrm{TM}}$ software, a proprietary software developed by Correlated Solutions, Inc. A description of the processing steps and techniques are described in the next section.

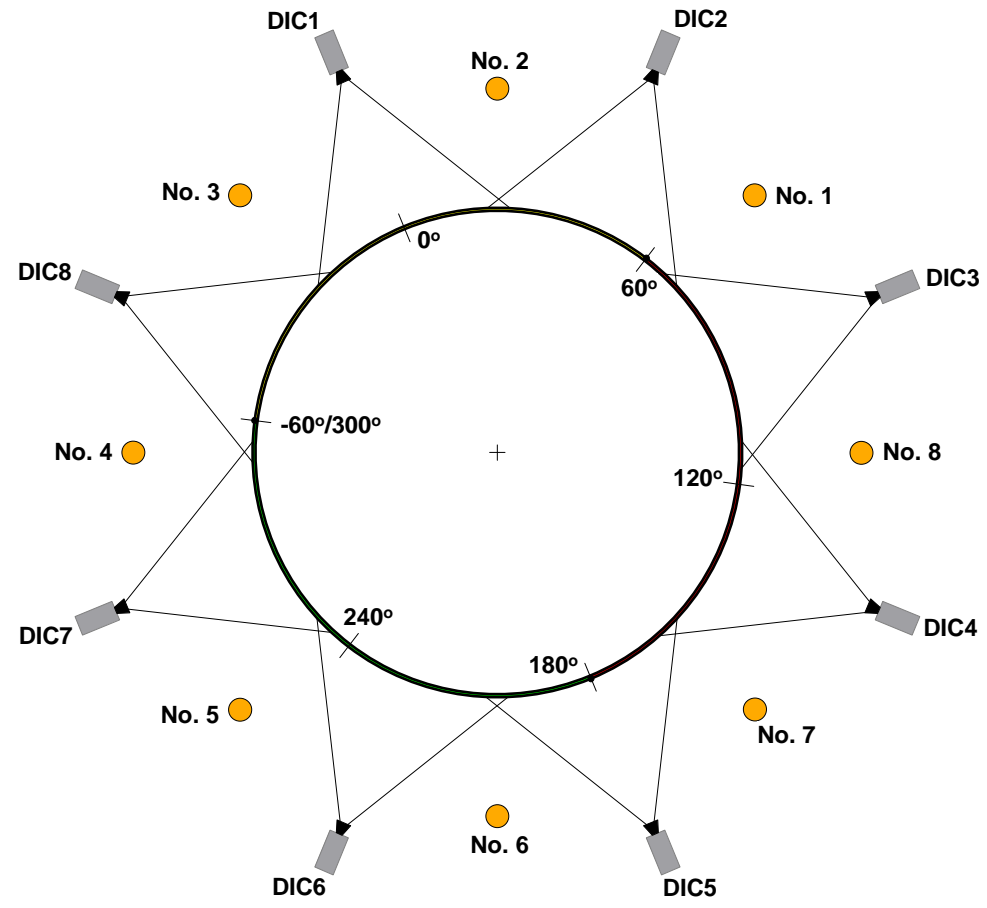

Figure 5. Low-speed DIC system layout.

\section{Digital Image Correlation Data Processing}

Data processing used built-in functions of VIC-3 $\mathrm{D}^{\mathrm{TM}}$, while data assembly and plotting was accomplished utilizing user-written scripts. A brief overview of the steps involved in the processing of the DIC images and data is presented in this section. Data processing and data transformation is presented in Section A, and data assembly and thresholding is presented in Section B. Refer to Ref. 3 for more information on DIC calibration and image correlation.

\section{A. Initial Processing and Data Transformation}

VIC-3 $\mathrm{D}^{\mathrm{TM}}$ was used to process the acquired DIC images for each system and calculate selected data. First, a reference image pair is selected that is associated with the unloaded and undeformed test article at the start of the test. Then, a region of the test article in the reference image is selected where the image correlation is to be performed, and referred to as the area of interest (AOI). A typical AOI is shown in Fig. 6(a) indicated by the purple shaded region. Next, the spatial resolution of the data is specified, i.e., the point spacing at which the data is evaluated and reported is defined. Image correlation is then performed between the reference image pair and subsequent image pairs over the

\footnotetext{
‡ This is not an endorsement by the National Aeronautics and Space Administration (NASA).
} 
entire AOI, i.e. correlation between the undeformed image and deformed images. The image correlation process calculates surface position coordinates and displacements, provided in Cartesian coordinates, and corresponding measurement uncertainties for each point within the AOI for each image pair.

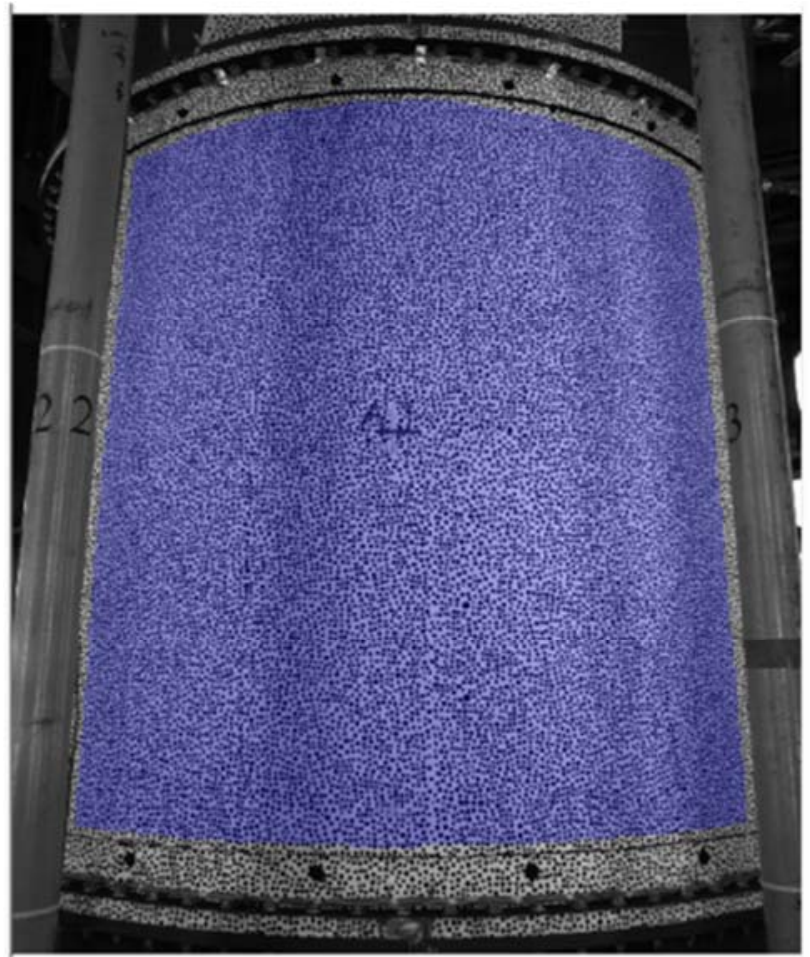

(a) Typical AOI

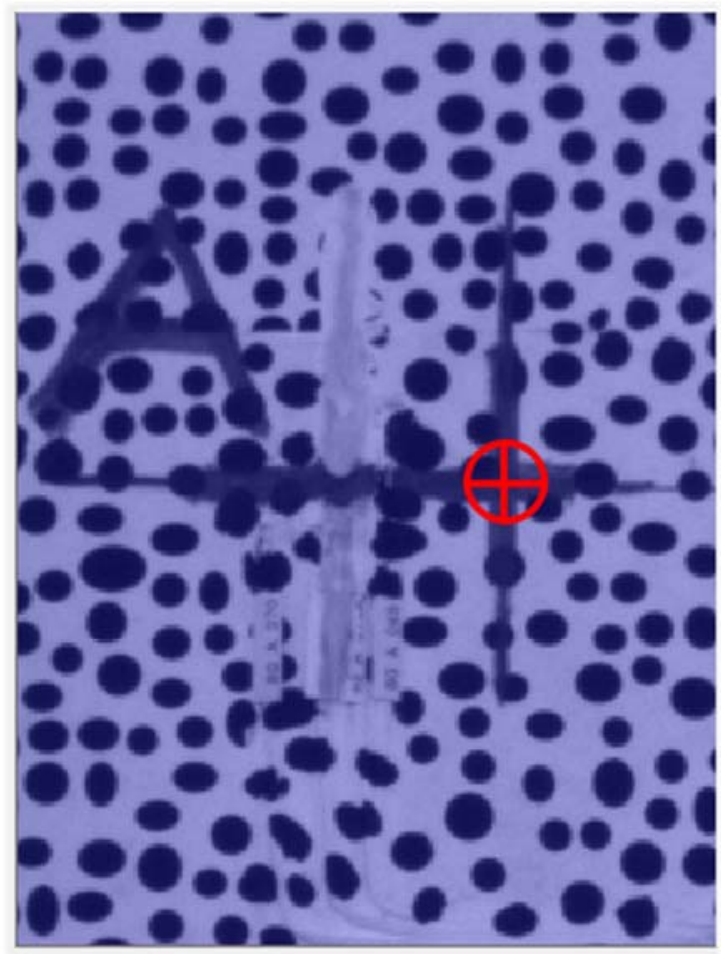

(b) Setting the origin at the center of Panel A

\section{Figure 6. Cylindrical transformation of DIC data using VIC-3D ${ }^{\mathrm{TM}}$}

After this initial processing was completed for each system, VIC-3D ${ }^{\mathrm{TM}}$ was used to calculate a best-fit radius, $R_{V I C}$, for each cylinder arc-section based on the DIC-measured surface geometry data from each system. The best fit radius for each system is summarized in Table 1 . It should be noted that the average $R_{V I C}$ for all eight systems was 47.888 inches, which is within $0.189 \%$ of the structured-light-based radius measurement of 47.979 inches. This bestfit radius was then used to transform the DIC data from a Cartesian coordinate system into a cylindrical coordinate system. The origin of the cylindrical coordinate system is defined using ' + ' markers included in the speckle pattern positioned at known axial and circumferential locations (e.g., see Fig. 6b)

Table 1. DIC cylindrical transformation data and values required for data assembly of ITA02.

\begin{tabular}{|c|c|c|c|c|c|}
\hline System & Best-fit Radius, $\boldsymbol{R}_{\text {VIC }}[\mathbf{i n}]$ & $\begin{array}{c}\text { Reference } \\
\text { Location }\end{array}$ & $\theta$-reference $\left[^{\circ}{ }^{]}\right.$ & \multicolumn{2}{|c|}{$\theta$ - thresholding $\left.{ }^{\circ}{ }\right]^{\prime}$} \\
\hline $\mathbf{1}$ & 48.151 & Center Panel A & 0 & -23 & 25 \\
\hline $\mathbf{2}$ & 47.748 & Weld A/B & 60 & 26 & 64 \\
\hline $\mathbf{3}$ & 47.809 & Weld A/B & 60 & 65 & 118 \\
\hline $\mathbf{4}$ & 47.768 & Center Panel B & 120 & 119 & 161 \\
\hline $\mathbf{5}$ & 48.104 & Weld B/C & 180 & 162 & 199 \\
\hline $\mathbf{6}$ & 47.894 & Center Panel C & 240 & 200 & 247 \\
\hline $\mathbf{7}$ & 47.666 & Weld C/A & 300 & 248 & 297 \\
\hline $\mathbf{8}$ & 47.965 & Weld C/A & 300 & 298 & -24 \\
\hline
\end{tabular}




\section{B. Data Assembly and Thresholding}

Once the data has been processed in VIC-3D ${ }^{\mathrm{TM}}$, user-written scripts were used to assemble the individual data sets into a single data set and remove overlapping data between adjacent DIC systems. First, the VIC-3D data was transformed into a global surface coordinate system with the axial and circumferential surface coordinates defined as $x$ and $\theta$, respectively, shown in Fig. 7. Specifically, the mid-length of the cylinder is set at $x=0$ and $\theta$ - reference locations, such as those shown for ITA02 in Table 1, were used to adjust the VIC-3D $\theta$-coordinate data from each DIC system into the proper global surface coordinate location. Next, the overlapping regions of data from each DIC system were removed before any further processing or data analysis was conducted by applying a simple data thresholding procedure. Here, the magnitude of the full-field measurement uncertainty, $\sigma_{\mathrm{mag}}$, was calculated by VIC3D for each measurement AOI. $\sigma_{\text {mag }}$ for a typical DIC system, shown in Fig. 8. The red/orange contours correspond to the maximum measurement uncertainty and the blue/purple contours correspond to the minimum measurement uncertainty. In general, the minimum uncertainty was found near the center of the correlated region, increasing to a maximum value approaching the edges of the region. Uncertainty data from each system was extracted at the midlength of the test article (indicated by the black dotted line in Fig. 8) and plotted, as shown in Fig. 9. The data thresholding locations were selected to correspond to the points of intersection between adjacent uncertainty curves, marked with gray filled circles in Fig. 9. The thresholding locations for each system, referred to as $\theta$ - thresholding, are summarized in Table 1. Once these thresholding points were identified, the DIC data outside these points was removed from the data set. In addition, an estimate of the displacement measurement uncertainty can be established. For this case, the maximum $\sigma_{\operatorname{mag}}$ is equal to approximately 0.001 . Thus, a $3 \sigma_{\mathrm{mag}}$ displacement uncertainty (corresponding to a $99.7 \%$ confidence interval) is approximately \pm 0.003 inch. The progression of developing a fullfield contour plot is shown in Fig. 10 using data thresholding (the individual DIC camera systems are separated by the solid vertical lines and numbered). By using this type of thresholding technique, the measurement uncertainty in the assembled data was minimized.

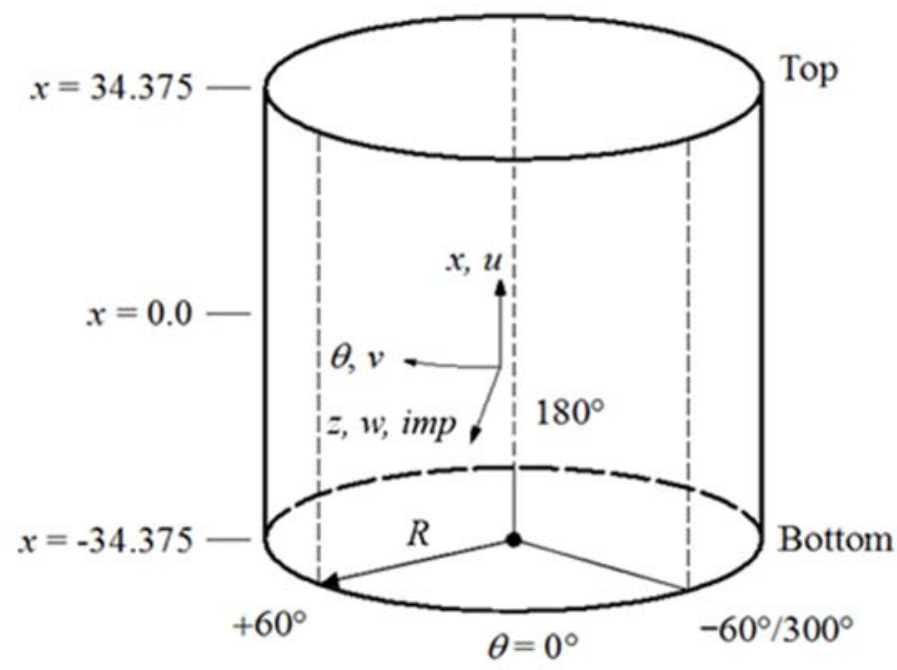

Figure 7. Global surface coordinate system and displacement definitions. The dashed lines indicate the location of longitudinal welds. (Axial dimensions are shown in inches) 
$\sigma_{\text {mag, }}$ inches

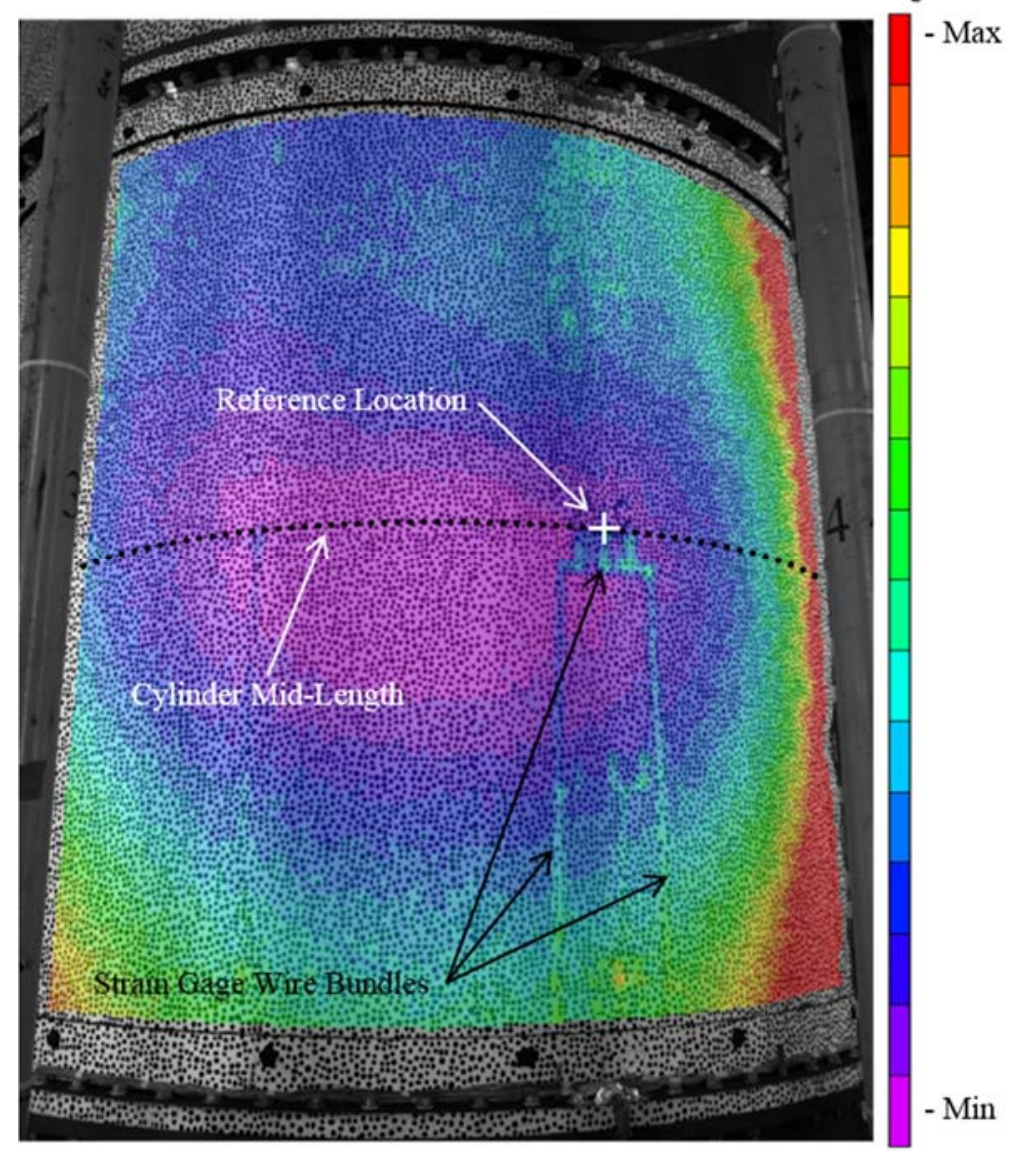

Figure 8. Magnitude of measurement uncertainty, $\sigma_{\text {mag, }}$ contour for a typical set-up and camera system.

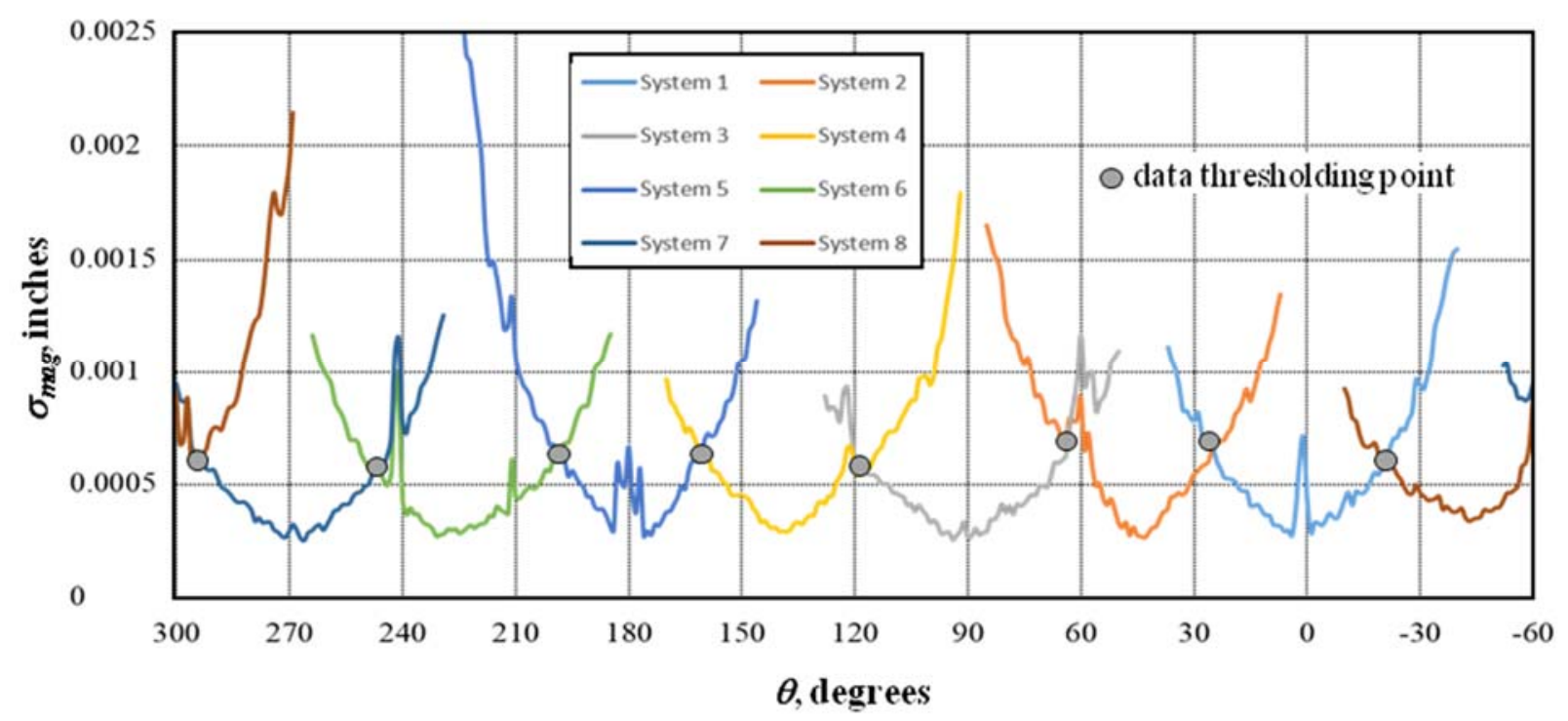

Figure 9. Magnitude of measurement uncertainty $\sigma_{\mathrm{mag}}$ at the mid-length of a test article. 


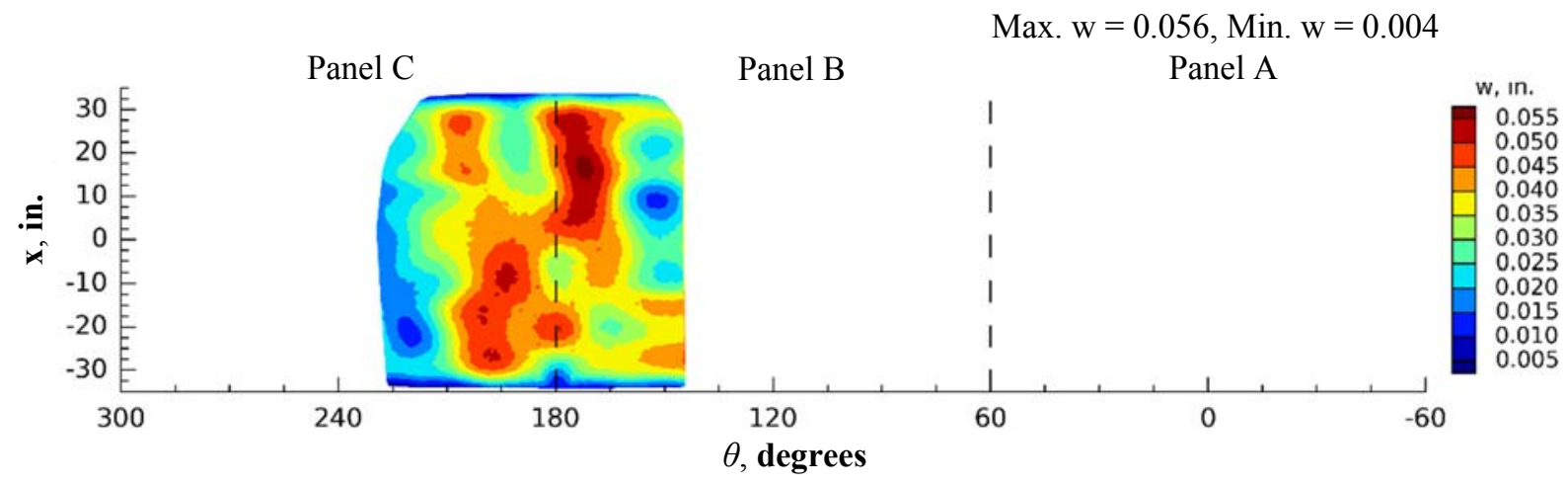

(a) Radial displacement contour plot for System 5 without data thresholding

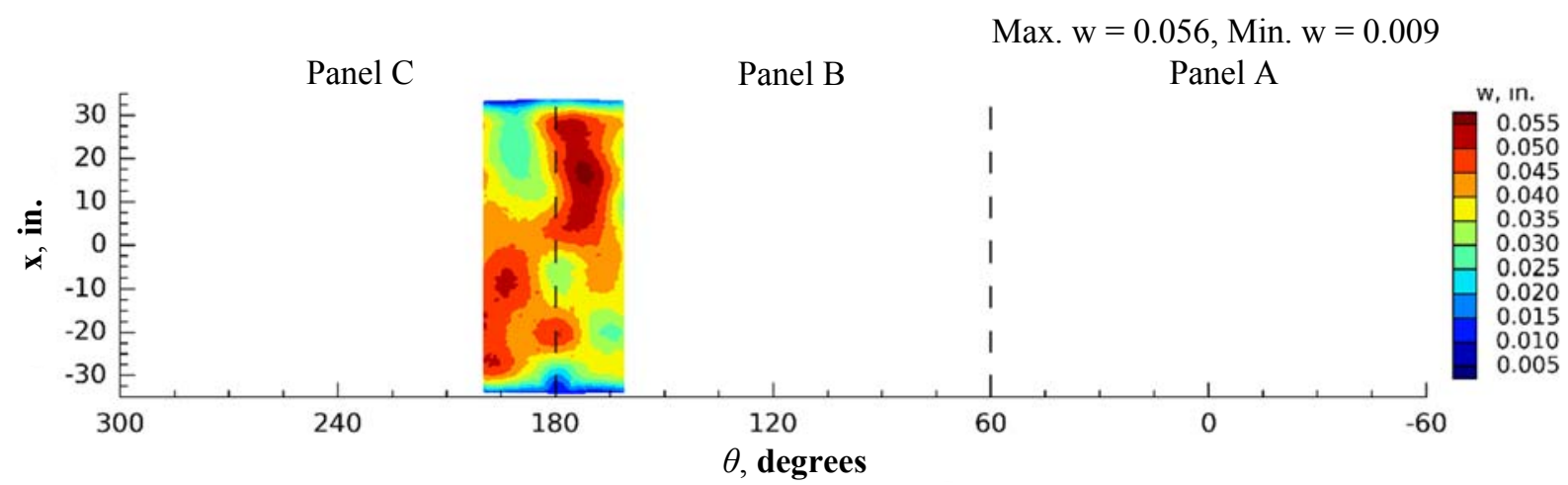

(b) Radial displacement contour plot for System 5 with data thresholding

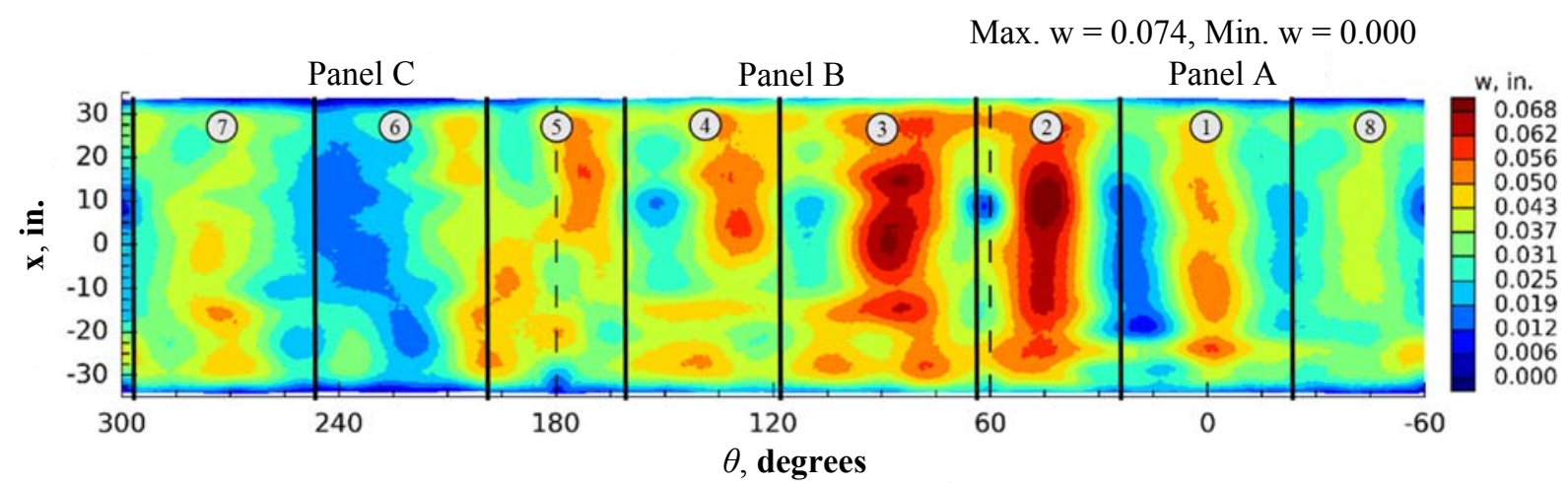

c) Radial displacement contour plot for all Systems with data thresholding

Figure 10. Typical radial displacement data assembly using data thresholding.

\section{Results and Discussion}

Full-field DIC data provides a significant amount of the displacement data necessary for the validation of highfidelity cylinder buckling simulations used by SBKF. In addition, DIC data has been used to verify measured data obtained from other instrumentation, such as LVDTs and electrical-resistance strain gages, and can help identify and characterize the root cause of anomalous behavior. Examples of using DIC data to verify measured cylinder geometry data, understand unexpected test results, and refine high-fidelity structural simulations are presented for two different compression-loaded 8-ft-diameter metallic cylindrical test articles.

\section{A. Initial Geometric Imperfection of Test Article - 07 (TA07)}

The response of compression-loaded thin-walled cylinders can be sensitive to initial geometric imperfections. ${ }^{4}$ In addition, the buckling response of these cylinders can be predicted accurately if the initial geometric imperfections are properly characterized and included in the model. ${ }^{2}$ Thus, prior to installation in the test facility, the as-built geometry of the test article's OML was measured using a photogrammetry/structured-light geometry measurement 
technique, with a measurement accuracy of \pm 0.001 inch. The deviation of the measured geometry from a best-fit circular cylinder was calculated and defined as the initial geometric imperfection, imp. However, it is possible that changes in the initial cylinder geometry may occur during its installation into the test facility. Therefore, the asinstalled initial geometric imperfection was measured using DIC, with a measurement accuracy of \pm 0.003 inch. This as-installed imperfection was then compared to the original measured imperfection to identify and characterize any changes in the geometry. A plot of the measured geometric imperfection obtained from the structured-light measurement technique is shown in Fig. 11a and the corresponding DIC-based initial geometric imperfection is shown in Fig. 11b. The DIC-based imperfection is based on the fully assembled DIC data set for the cylinder and was defined as the difference between a best-fit radius, $R_{D I C}$, and the measured radius at each point, $R_{\text {measured }}$, that is $i m p=R_{\text {measured }}$ $-R_{D I C}$. Next, the calculated imperfections were fit with a Fourier series of the form given by

$$
i m p=\sum_{i=0}^{n} \sum_{j=0}^{m} \cos \left(i \pi \frac{x}{L}\right)\left(A_{i j} \cos \left(j \frac{\theta \pi}{2}\right)+B_{i j} \sin \left(j \frac{\theta \pi}{2}\right)\right)
$$

where $x$ is the axial coordinate, $L$ is the cylinder length, $\theta$ is the circumferential coordinate, and $A_{i j}$ and $B_{i j}$ are the Fourier coefficients. The Fourier series representation was used to smooth out some of the surface irregularities in the measured imperfection associated with the strain gage wires and discontinuities in the measured data between adjacent systems that typically occur when the data is merged together. The Fourier series fit used 15 axial half-waves $(n=15)$ and 30 circumferential waves $(m=30)$. The overall character of the two measured imperfections was similar, although slight differences in the imperfection distribution can be seen. By subtracting the initial geometric imperfection in Fig. 11a from Fig. 11b, an imperfection difference plot, referred to herein as a $\Delta i m p$ plot, was generated in an effort to identify any slight changes in the initial surface geometry that occurred during the installation of the cylinder into the test facility (shown in Fig. 11c). The results indicate that the initial geometry had changed in some places by more than $+/-0.050 \mathrm{inch}$. In addition, the imperfection amplitude at the weld lands decreased by approximately $0.010-0.020$ inch. A version of this DIC-based imperfection was included in a high-fidelity finite-element model of TA07 and resulted in a $4.7 \%$ increase in buckling load and a substantial improvement in the overall correlation with the test results. More details on this model improvement are provided in Ref. 2.
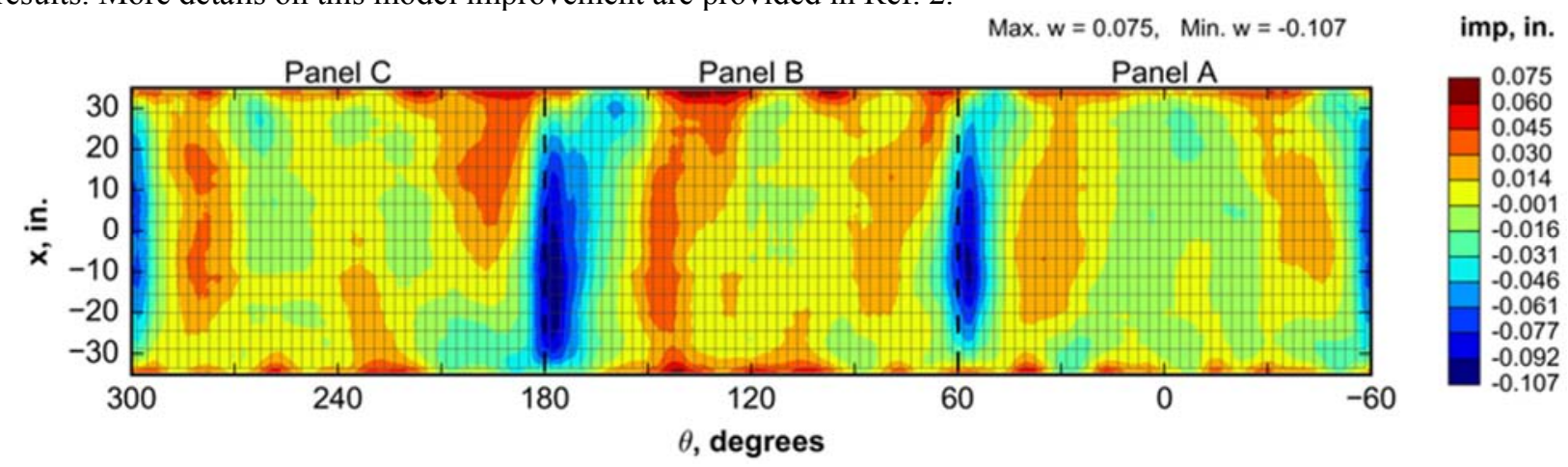

a) Structured-light-based initial geometric imperfection for TA07

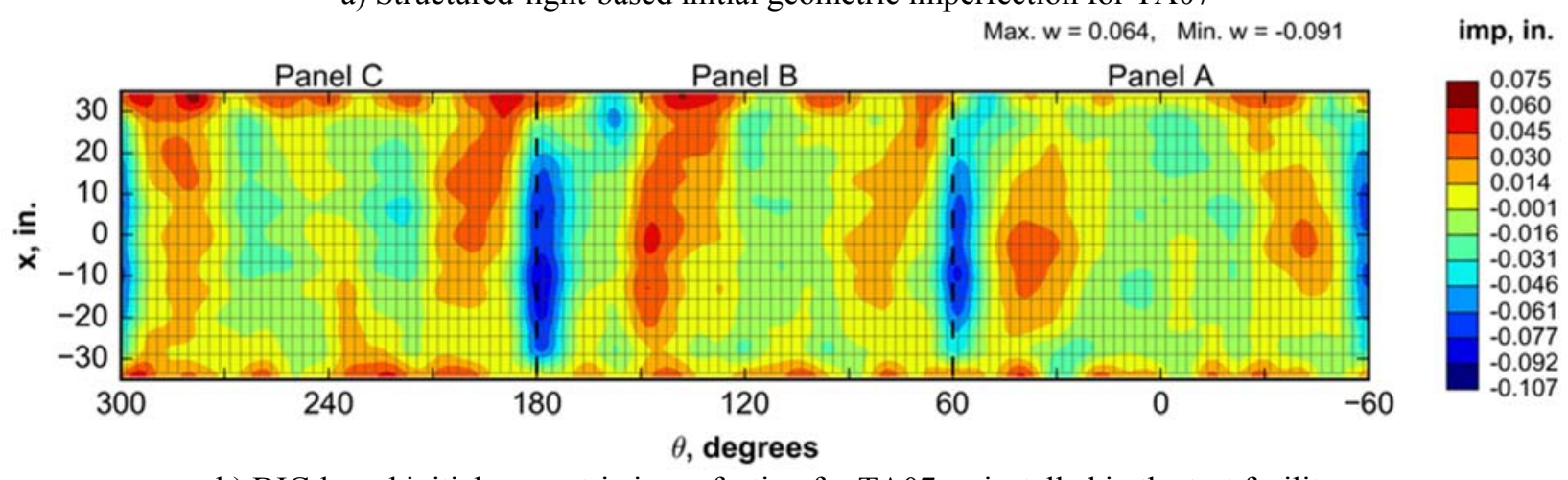

b) DIC-based initial geometric imperfection for TA07 as-installed in the test facility

Figure 11. DIC-based measured geometric imperfection and imperfection delta plot for TA07 


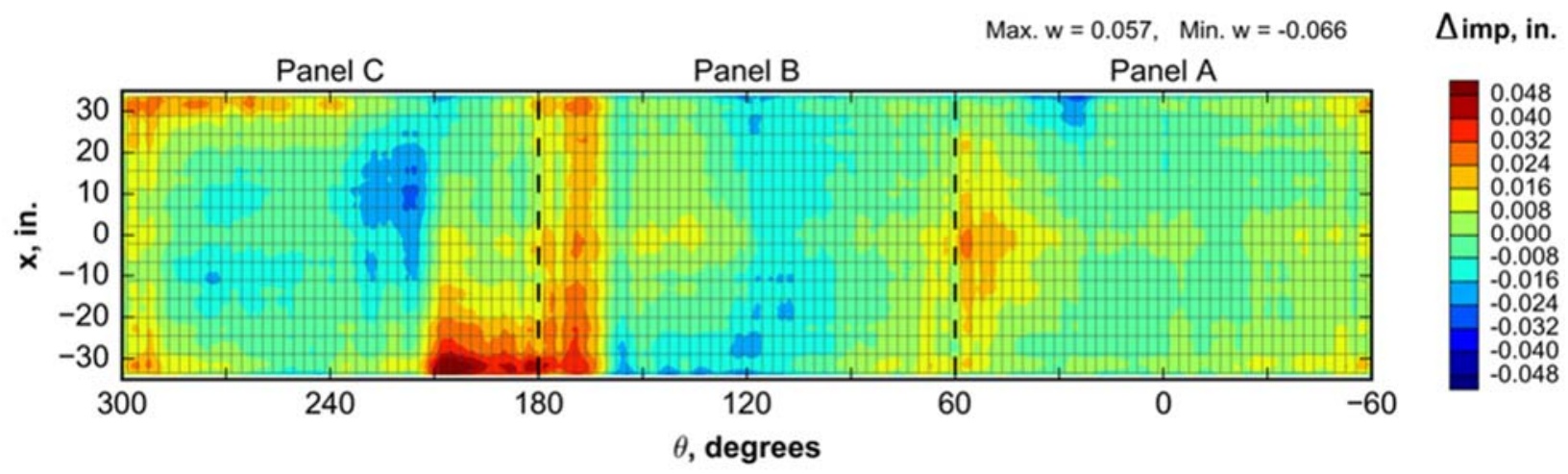

c) $\Delta$ imp plot between the structured-light-based geometric imperfection and the DIC-based geometric imperfection

Figure 11. DIC-based measured geometric imperfection and imperfection delta plot for TA07 (continued).

\section{B. Characterizing Anomalous Behavior of Isogrid Test Article - 02 (ITA02)}

ITA02 was tested to buckling failure under uniform axial compression. During the test, some anomalous data trends were observed with one particular observation described here. The measured load versus radial displacement response at the center of one of the longitudinal welds in ITA02 is shown in Fig. 12, indicated by the solid red line. ITA02 exhibits a monotonically increasing response up to a load of $709 \mathrm{kips}$, at which point the test load sequence entered into a pre-defined hold condition (in this case, a pause in loading in order to assess measured data). During this hold, the LVDT displacement data slowly increased from approximately 0.030 inches to 0.037 inches while the load control system maintained a constant applied load of 709 kips, represented by the data between the two gray filled circles. Previous testing exhibited similar discontinuities in the measured displacement data, but were attributed to issues with LVDT instrumentation. In this case, however, other LVDTs and strain gages also indicated shifts in the data during the load hold, thus further investigation was conducted to identify the root cause of this anomalous behavior and is described here.

First, LVDT radial displacement data was compared to DIC-based measurements, indicated by the red circles in Fig. 12. The DIC data exhibits a similar shift in the displacement response during the load hold, thus verifying the anomalous event existed in the test article and was not an issue the LVDT measurement. In fact, additional shifts in radial displacement can be seen to occur at other load levels during the load sequence.

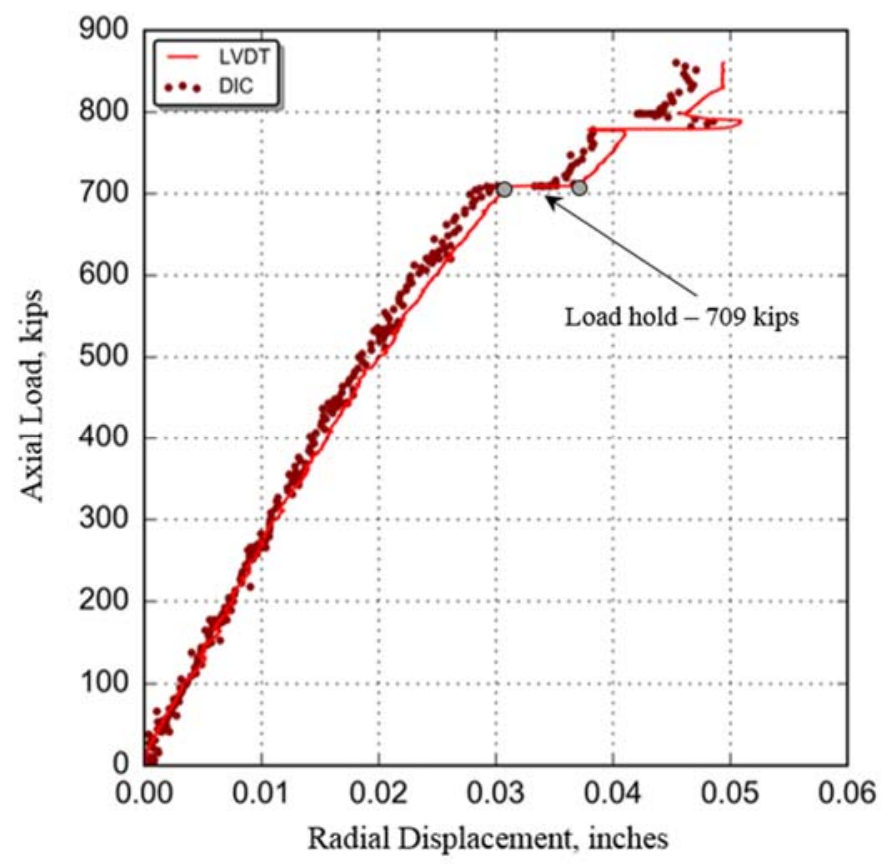

Figure 12. Load versus radial displacement response at weld land $B / C\left(\theta=180^{\circ}\right)$. 
Next, selected DIC radial displacement contours from ITA02 at the beginning and end of the load hold were plotted and compared in an attempt to characterize the anomalous displacement response. Radial displacement contours at the beginning and at the end of the load hold are shown in Figs. 13a and 13b. Under close inspection, slight changes in the contour pattern are observed at the $\theta=60^{\circ}$ and $\theta=180^{\circ}$ circumferential locations (weld land $\mathrm{A} / \mathrm{B}$ and $\mathrm{B} / \mathrm{C}$, respectively), while the overall character of the displacement response appears similar throughout the hold. A $\Delta w$ plot (change in radial displacement) was created by subtracting the radial displacements of Fig. 13a from Fig. 13b. The resulting $\Delta w$ plot is shown in Fig. 13c and indicates two distinct regions of ITA02 undergoing a change in the radial displacement response along two of the three axial welds. The corresponding $\Delta u$ plot (change in axial displacement) is shown in Fig. 14 and suggests that this displacement response was a result of some localized movement or settling of ITA02 at the upper and lower potted boundaries (see regions outlined with dashed ovals). Finally, circumferential traces of the $\Delta u$ response at the upper and lower boundary of the cylinder were obtained from the contour data in Fig. 14 and are shown in Fig. 15, indicated by the blue (upper boundary) and orange (lower boundary) curves, respectively. The $\Delta u$ data shows an axial displacement at the boundary of ITA02 of approximately 0.0015 inches spanning a $30^{\circ}$ arc-section corresponding to movement away from the mid-length of the cylinder, i.e. the cylinder appears to be moving or settling into the attachment rings. It should be noted that even though these $\Delta u$ displacement results were less than the accuracy of our DIC measurements [ \pm 0.003 inches], it is evident from the $\Delta u$ and $\Delta w$ plots, Figs. 13 and 14, and the $\Delta u$ line circumferential traces as shown in Fig. 15, that this behavior had occurred

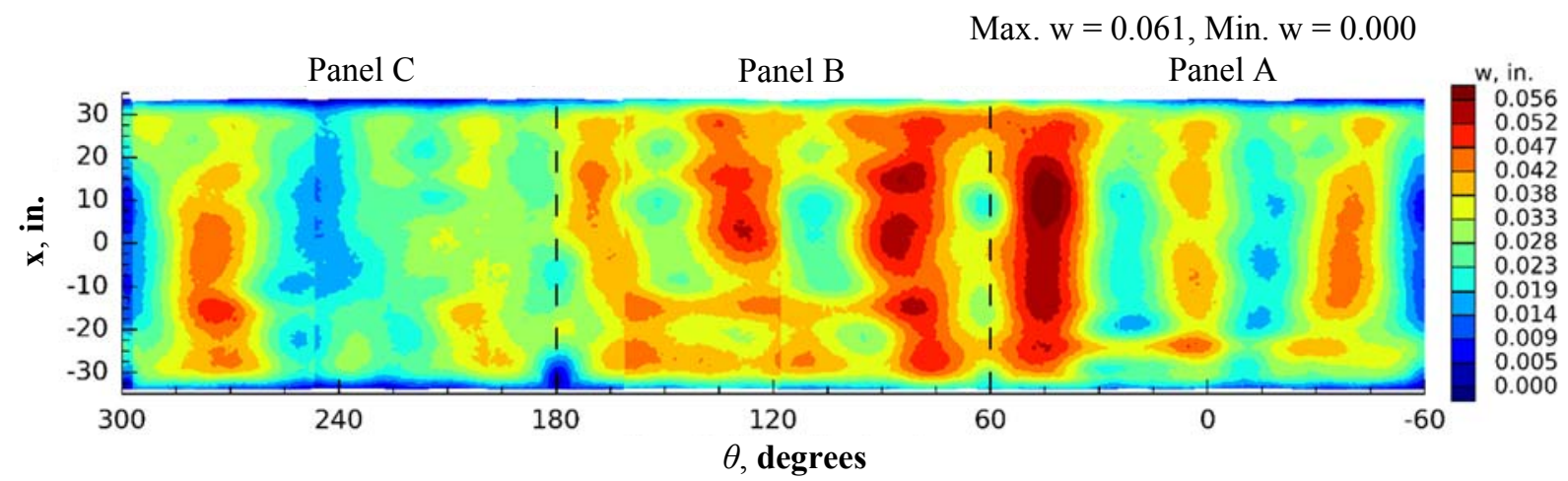

(a) Beginning of hold

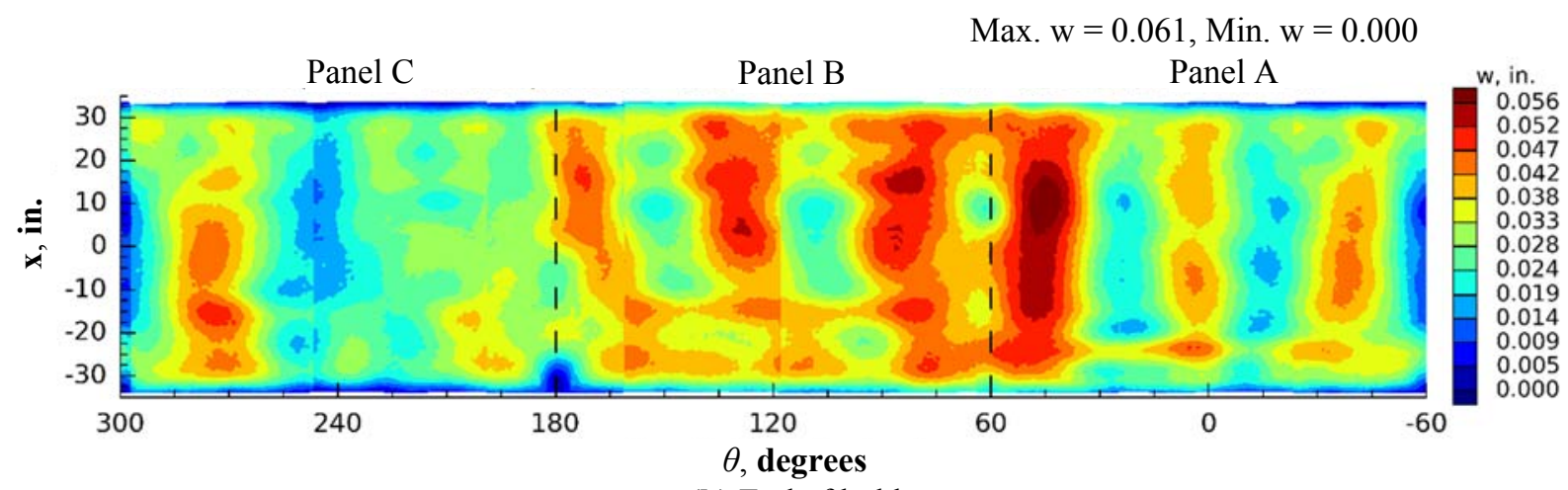

(b) End of hold

Figure 13. Contour plots showing displacements occurring in ITA02 during a hold in loading at 709 kips. 


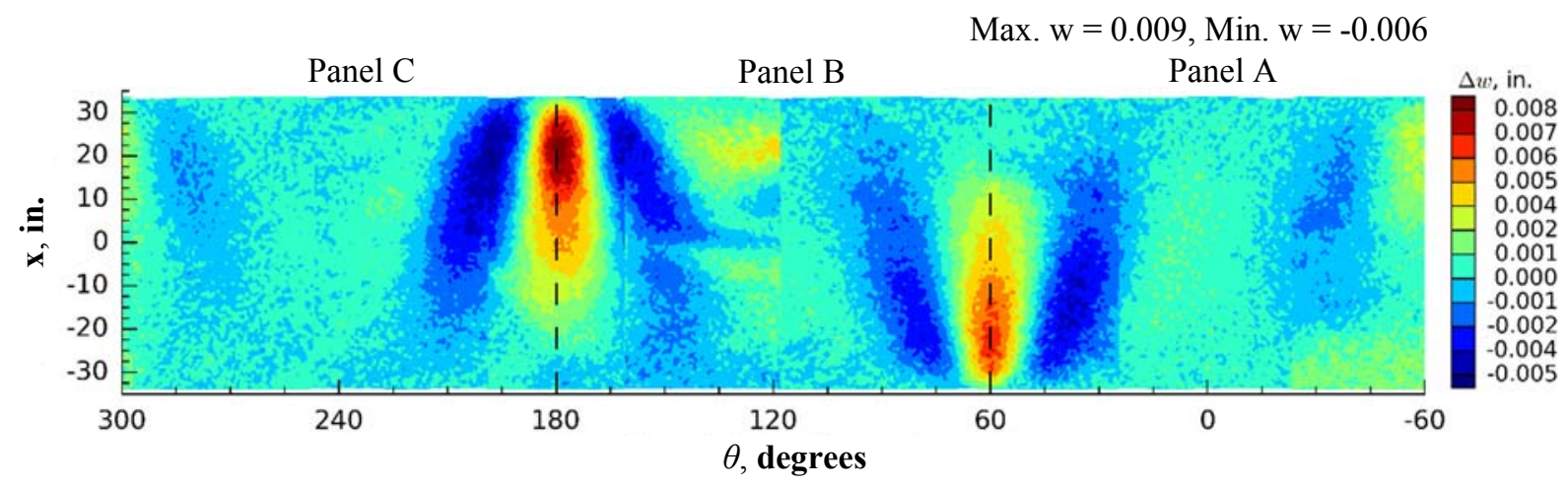

(c) Change in radial displacment during load hold - $\Delta w$

Figure 13. Contour plots showing displacements occurring in ITA02 during a hold in loading at 709 kips (continued).

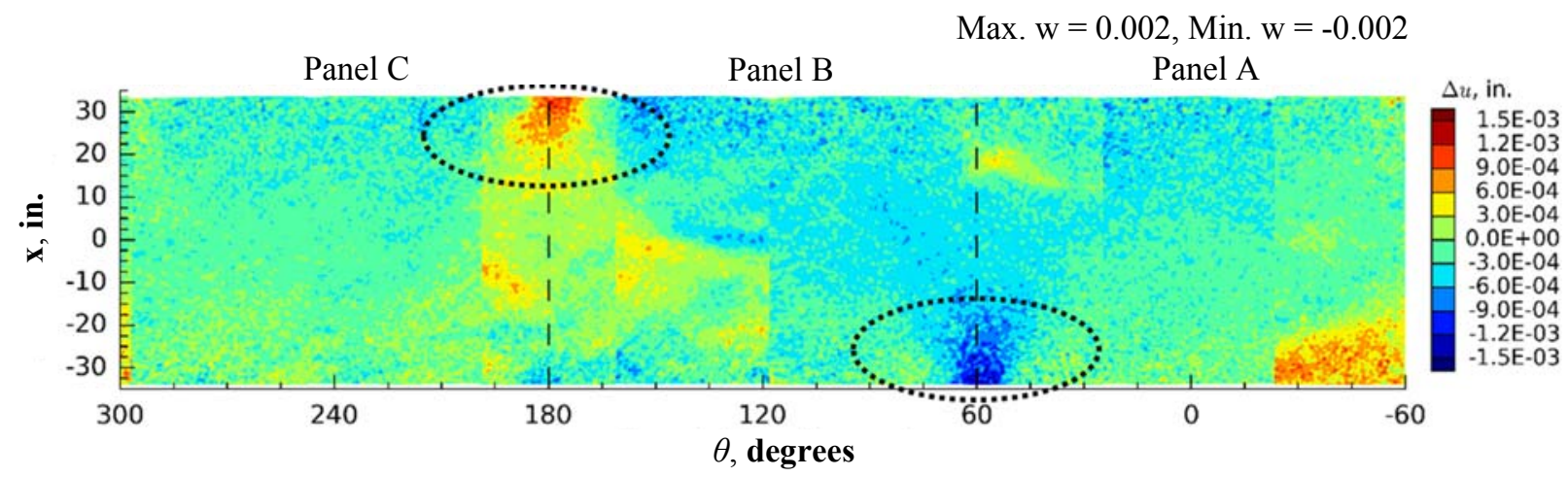

Figure 14. Change in axial displacement during load step 7 hold - $\Delta u$.

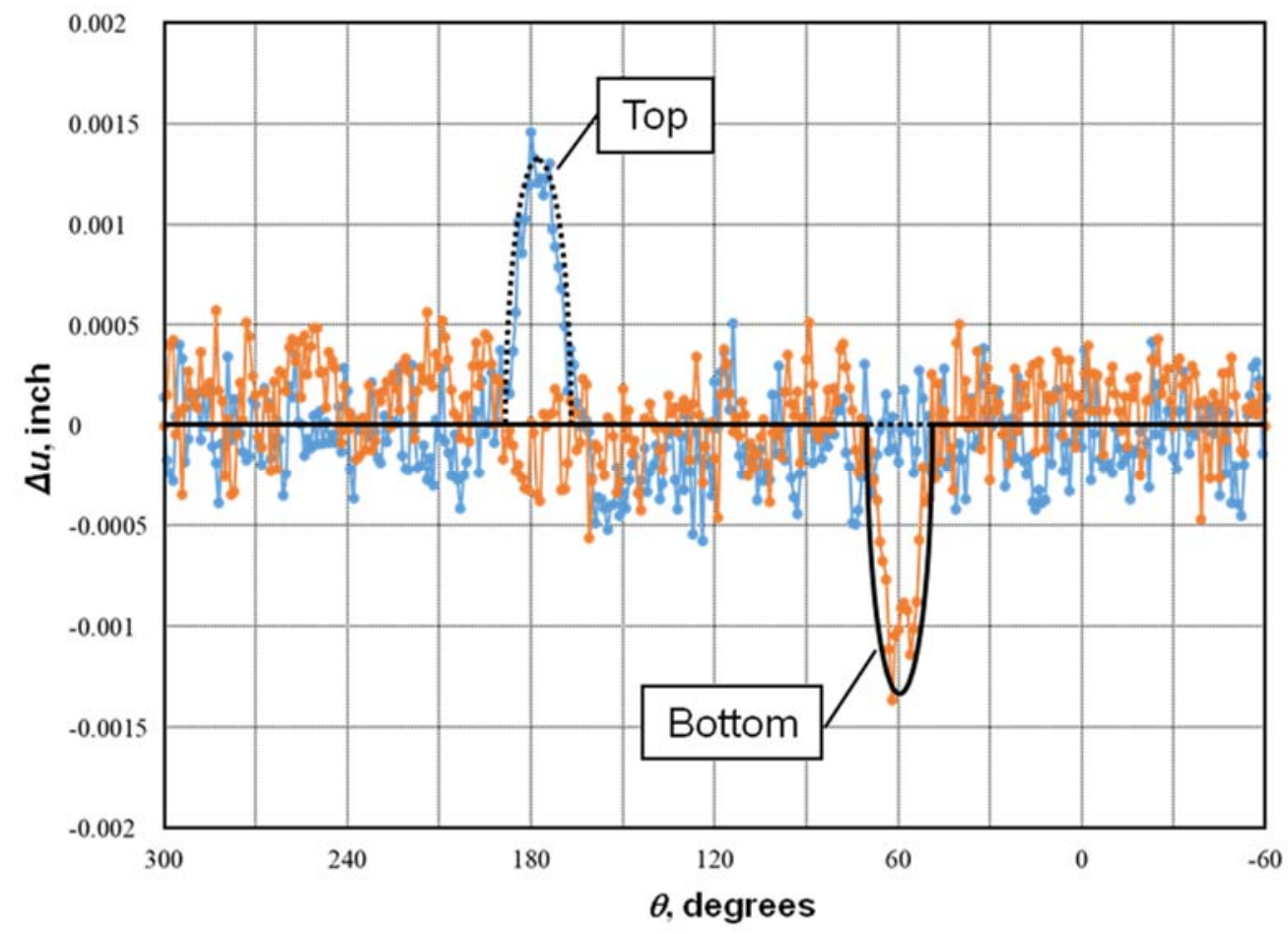

Figure 15. Measured $\Delta u$ displacement data at the top and bottom boundary of ITA02. 
The final step in the investigation of the anomalous ITA02 behavior was to simulate the effects of the $\Delta u$ displacements and compare the predicted $\Delta w$ response with the measured response shown in Fig. 13c. A best-fit version of the $\Delta u$ data from Fig. 15 (indicated by the black solid and dashed curves) was incorporated to a finiteelement model of ITA02 to simulate the settling of the cylinder boundary during the load hold described in Section IV-B. The ITA02 model was first loaded in compression to a load level of 709 kips by applying a uniform end displacement. Then, the $\Delta u$ displacements were added to the applied displacements to simulate the settling of the cylinder in the attachment rings during the load hold. Finally, additional axial load was applied to the cylinder up to the buckling load. The predicted $\Delta w$ displacement contour during the simulated load hold is shown in Fig. 16, and the predicted and measured load versus radial displacement data from the center of weld land $\mathrm{B} / \mathrm{C}\left(\theta=180^{\circ}\right)$ are shown in Fig. 17. The measured results plotted in Fig. 17 are the same LVDT results shown in Fig. 12 (indicated by the red solid line) and the predicted results include results from a finite-element model with and without the $\Delta u$ displacements applied indicated by the green and blue solid lines, respectively. These results shown in Figs. 16 and 17 indicate that a model that includes the $\Delta u$ displacement can accurately predict the anomalous displacement response observed in ITA02, providing conclusive evidence that settling events in the potted boundaries of ITA02 resulted in the unexpected LVDT displacement readings.

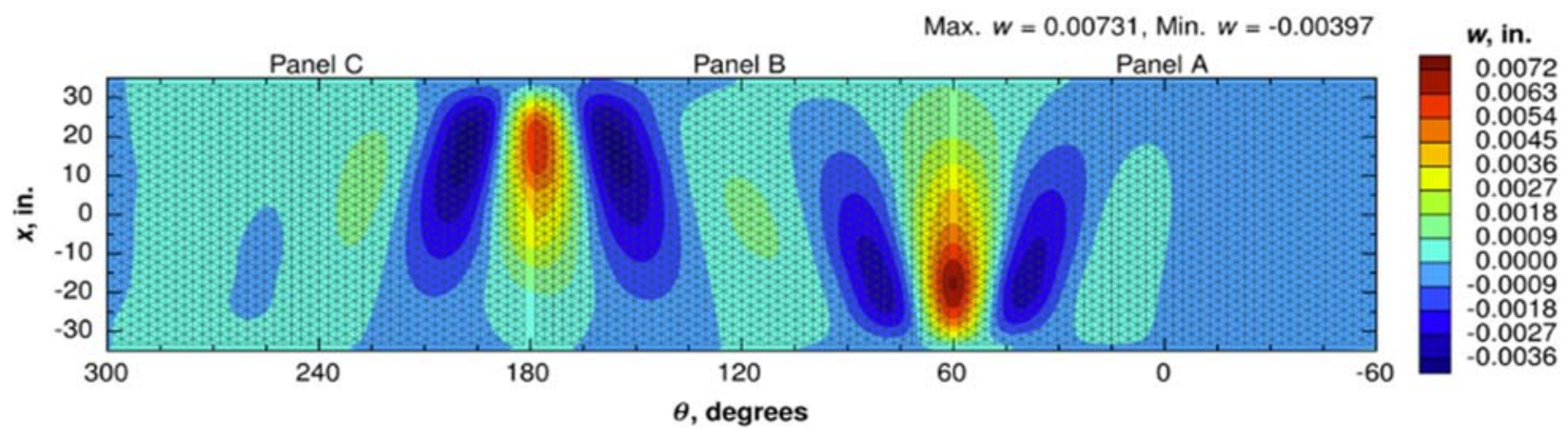

Figure 16. Predicted $\Delta w$ displacement response during simulated settling event.

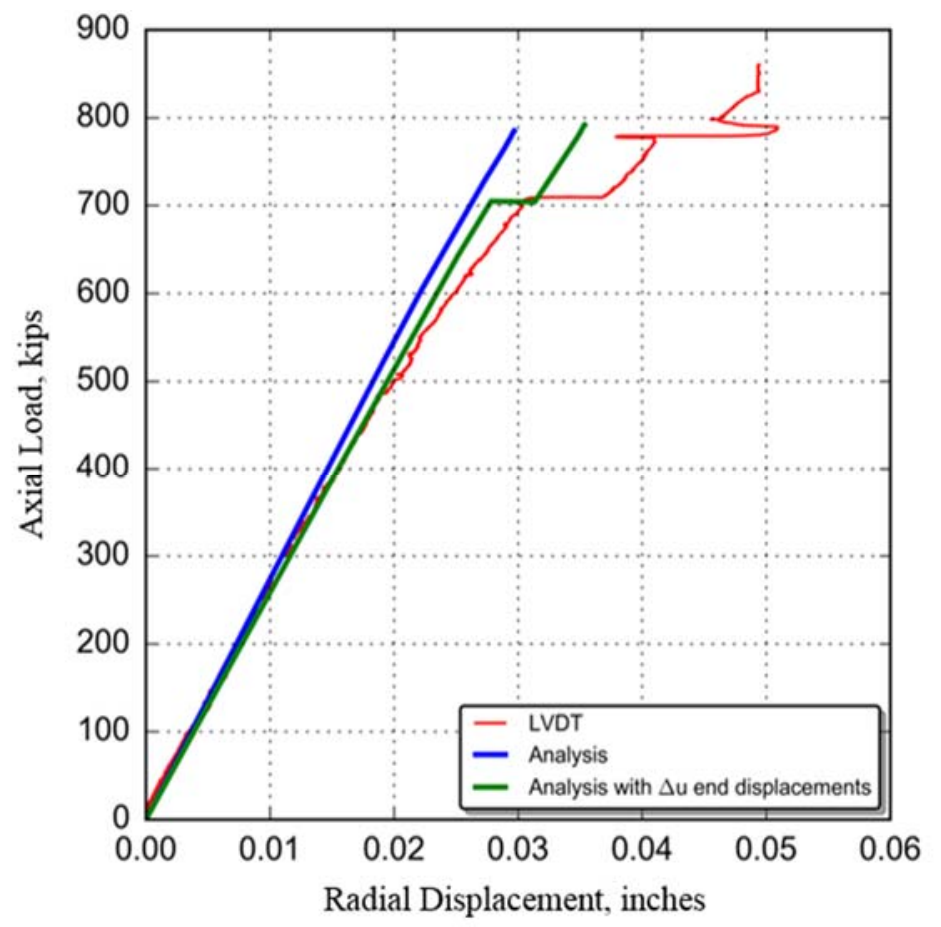

Figure 17. Predicted and measured load versus radial displacement response at weld B/C. 


\section{Concluding Remarks}

DIC was successfully used on a series of subscale cylinder tests to gather full-field displacement and strain data needed to validate high-fidelity cylinder buckling simulations and new analysis-based buckling knockdown factors. Selected DIC results from two different subscale metallic cylindrical test articles were presented to illustrate the application of DIC in large-scale structural testing and finite-element-model development. First, a detailed description of some of the DIC data processing methods were presented and included discussions on data assembly and measurement error estimation. Next, DIC data was presented and used to identify and characterize changes in the geometry of one cylinder test article TA07 that resulted from the installation of the test article into the test facility. Using this DIC data, an updated high-fidelity finite-element model of TA07 was created to account for the as-installed cylinder geometry and helped improve existing buckling predictions. Finally, DIC displacement data from a second cylinder test ITA02 was presented, and used to identify and characterize the root cause of anomalous LVDT displacement measurements observed during testing. The DIC data indicated that the anomalous LVDT response coincided with a local settling of the test article into the potted attachment rings of approximately $0.0015 \mathrm{inch}$. This DIC data was included in a finite-element model to simulate the settling of the test article, and the anomalous LVDT measurements were replicated.

\section{Acknowledgements}

This work was conducted as part of the NASA Engineering and Safety Center Shell Buckling Knockdown Factor Project, NESC Assessment \#:07-010-E. The large-scale testing was performed by the Structural Strength Test Group (ET30) at NASA Marshall Space Flight Center (MSFC) in Huntsville, Alabama.

\section{References}

${ }^{1}$ Hilburger M.W., "Developing the Next Generation Shell Buckling Design Factors and Technologies," Proceedings of the 53rd AIAA/ASME/ASCE/AHS/ASC Structures, Structural Dynamics and Materials Conference, AIAA paper no. 2012-1686, Honolulu, HI, April 2012.

${ }^{2}$ Hilburger M.W., Lindell, M. C., Waters, W. A., and Gardner, N. W., "Test and Analysis of Buckling-Critical Stiffened Metallic Launch Vehicle Cylinders," Proceedings of the 59th AIAA/ASME/ASCE/AHS/ASC Structures, Structural Dynamics and Materials Conference, Kissimmee, FL, 2018 (submitted for publication).

${ }^{3}$ Sutton, M. A., Orteau, J.-J., and Schrier, H. W., Image Correlation for Shape, Motion, and Deformation Measurements, Springer Science + Business Media, New York, 2009 pp.113-117.

${ }^{4}$ Hilburger, M. W., Starnes, J. H., Jr., "Effects of Imperfections on the Buckling Response of Compression-Loaded Composite Shells," International Journal of Non-Linear Mechanics, vol. 37, no. 4-5, pp. 623-643, 2002. 